\title{
Experimental Pathology
}

National Cancer Institute

\section{Source}

National Cancer Institute. Experimental Pathology. NCI Thesaurus. Code C19164.

Experimental pathology; the study of disease processes by the manipulation of the natural state of an organism. 